\title{
Atividade antioxidante e teor de fenólicos totais em hortaliças orgânicas e convencionais
}

\author{
Antioxidant activity and phenolic content in organic and conventional vegetables
}

\author{
Kettelin Aparecida ARBOS ${ }^{1 \star}$, Renato João Sossela de FREITAS ${ }^{2}$, \\ Sônia Cachoeira STERTZ2 ${ }^{2}$, Marlos Ferreira DORNAS ${ }^{3}$
}

\begin{abstract}
Resumo
Considerando que os consumidores estão mais conscientes com a sua saúde e mais preocupados com as condições ambientais, verifica-se uma crescente demanda por alimentos orgânicos. O objetivo deste estudo foi comparar a atividade antioxidante e o teor de fenólicos totais entre alface, rúcula e almeirão de cultivos orgânico e convencional, plantados em uma mesma horta. A atividade antioxidante dos extratos metanólicos das hortaliças foi determinada através do ensaio do DPPH. O teor de fenólicos totais foi obtido espectrofotometricamente de acordo com o método de Folin-Ciocalteu e calculado como equivalente de ácido gálico. A atividade antioxidante contra o radical livre DPPH foi: rúcula orgânica > almeirão orgânico > alface orgânica > rúcula convencional > almeirão convencional > alface convencional. O teor de fenólicos totais foi superior nos vegetais orgânicos. Os resultados deste estudo sugerem que as hortaliças orgânicas podem ser uma importante fonte de antioxidantes naturais e de compostos fenólicos e, portanto, seu consumo deve ser estimulado.

Palavras-chave: hortaliças; ação antioxidante; sistema de produção; DPPH; fenólicos.
\end{abstract}

\begin{abstract}
Considering that consumers are becoming more conscious of their health and more concerned with environmental conditions, there has been an increasing demand for organic foods. The aim of this study was to compare the antioxidant activity and determine the total phenolic content of organic and conventional cultivations of lettuce, arugula, and chicory grown in the same vegetable garden. The antioxidant activity of the methanol extracts of these leaf vegetables wasdetermined using DPPH free radical scavenging assay. The total phenolic content in the extracts was determined spectrometrically according to the Folin-Ciocalteu method and calculated as equivalent of gallic acid. The antioxidant activity against the DPPH free radical was: organic arugula $>$ organic chicory $>$ organic lettuce $>$ conventional arugula $>$ conventional chicory $>$ conventional lettuce. The total phenolic content was higher in the organic vegetables. The results of this study suggest that the organic vegetable can be a good dietary source of natural antioxidant and phenolic compounds; therefore, their consumption must be encouraged. Keywords: vegetables; antioxidant action; production system; DPPH; phenolic.
\end{abstract}

\section{Introdução}

O sistema de produção denominado atualmente de convencional baseia-se na utilização intensiva de insumos químicos (agrotóxicos), mecanização pesada e melhoramento genético voltado para a produtividade física. No entanto, esse padrão de produção visando exclusivamente à produtividade, vem sendo muito questionado, em função da divulgação de aspectos negativos, tais como esgotamento dos recursos naturais, degradação ambiental, exclusão social, elevação dos custos de produção, contaminação dos alimentos por agrotóxicos e redução de sua qualidade (PRIMAVESI, 1988; ORMOND et al., 2002; STAUB, 2003; CAMPOS, 2005).

Uma consequência da divulgação e da percepção desses aspectos indesejáveis tem sido a busca dos consumidores por dietas mais saudáveis e sem riscos para a saúde, materializada no crescimento da produção de alimentos orgânicos,

principalmente a partir da década de 90 (PENTEADO, 2000; MACHADO; CORAZZA, 2004). Contudo, embora crescente, a área agriculturável destinada à produção de orgânicos é estimada em apenas $0,25 \%$ da brasileira (BUAINAIN; BATALHA, 2007).

Em relação aos atributos de qualidade dos alimentos orgânicos, os resultados ainda são pouco conclusivos. Darolt (2003a) afirma existir um grande número de fatores que pode afetar a qualidade de um alimento, como fatores genéticos (variedades), clima, condições de solo, armazenamento póscolheita e modo de produção (orgânico ou convencional).

É indiscutível a ação benéfica que o consumo regular de frutas, verduras e hortaliças proporciona à saúde do homem. Estudos epidemiológicos têm demonstrado que o efeito protetor exercido pelo consumo desses alimentos se deve à presença

Recebido para publicação em 22/7/2008

Aceito para publicação em 16/5/2009 (003700)

${ }^{1}$ Programa de Pós-Graduação em Tecnologia de Alimentos, Universidade Federal do Paraná - UFPR, Centro de Ciência e Tecnologia de Alimentos, Universidade Anhanguera UNIDERP, Rua Alexandre Herculano, 1400, Jardim Veraneio, CEP 79480-000, Campo Grande - MS, Brasil, E-mail: kettelin.arbos@gmail.com

2 Programa de Pós-Graduação em Tecnologia de Alimentos, Centro Politécnico, Universidade Federal do Paraná - UFPR, CEP 81531-990, Curitiba - PR, Brasil

${ }^{3}$ Universidade para o Desenvolvimento do Estado e da Região do Pantanal - UNIVERSIA, Campo Grande - MS, Brasil

${ }^{*}$ A quem a correspondência deve ser enviada 
de componentes bioativos como a vitamina C, vitamina $\mathrm{E}$ e fitoquímicos com ação antioxidante, dentre os quais se destacam os compostos fenólicos, $\beta$-caroteno e vários outros carotenoides (RICE-EVANS; MILLER; PAGANGA, 1996; WANG; CAO; PRIOR, 1996; VELIOGLU et al., 1998; VISON et al., 1998; MELO et al., 2006).

A eficácia da ação antioxidante dos componentes bioativos depende da estrutura química e da sua concentração no alimento. Por sua vez, o teor de fitoquímicos em hortaliças é amplamente influenciado por fatores genéticos, condições ambientais, sistema de produção, além do grau de maturação e variedade da planta, entre outros (KAHKONEN et al., 1999; KAUR; KAPOOR, 2001; KOLEVA et al., 2002; MELO et al., 2006).

Numerosos estudos já foram conduzidos abordando as propriedades antioxidantes de frutas e hortaliças, e plantas medicinais (RAMOS et al., 2003; CAI et al., 2004; MIGUEL et al., 2004; SCHAFFER; SCHMITT-SCHILLIG; ECKERT, 2005; HSU; CHAN; CHANG, 2007; YU et al., 2007). Todavia, poucos trabalhos são conduzidos visando verificar a influência do tipo de cultivo na ação antioxidante desses alimentos. $\mathrm{O}$ que se pode verificar nesses estudos é que os alimentos produzidos organicamente têm tendência a possuírem menor teor de nitrato, maior teor de vitamina $\mathrm{C}$ e matéria seca, bem como maior teor de compostos com ação antioxidante, tais como flavonoides e carotenoides (WORTHINGTON, 2001; BOURN; PRESCOTT, 2002; WILLIAMS, 2002; DAROLT, 2003b; STERTZ; ROSA; FREITAS, 2005; MAGKOS; ARVANITI; ZAMPELAS, 2006).

No presente trabalho foram comparados a atividade antioxidante e o teor de compostos fenólicos de alface, rúcula e almeirão, obtidos de cultivos orgânico e convencional.

\section{Material e métodos}

\subsection{Cultivo das amostras}

As amostras de alface, rúcula e almeirão analisadas neste estudo foram provenientes de um mesmo lote de sementes, germinadas concomitantemente e cultivadas em canteiros orgânicos e convencionais. Dessa forma, alguns condicionantes que poderiam afetar a qualidade nutricional dessas hortaliças e, consequentemente, influenciar na ação antioxidante puderam ser minimizados, uma vez que as condições climáticas, variedade, irrigação, tempo de maturação e condições de armazenamento foram as mesmas para as hortaliças cultivadas orgânica e convencionalmente.

Sementes peletizadas de alface (Lactuca sativa) cultivar Verônica (AF 257), rúcula (Eruca sativa) e almeirão (Cichorium intybus L.) foram semeadas em bandejas com 128 células, sob cultivo protegido em estrutura fechada com tela. A semeadura constou de duas ou quatro sementes por célula, sendo nessa fase empregado substrato orgânico produzido no local.

Após aproximadamente 15 dias da germinação, as mudas foram transplantadas para o solo: metade das mudas foi plantada na horta orgânica e metade na horta convencional. O sistema de irrigação utilizado foi do tipo gotejamento, sendo aplicados turnos de rega diários de acordo com as necessidades das espécies cultivadas.

No sistema orgânico de produção, a adubação foi composta por biofertilizante preparado por meio da fermentação, contendo esterco de bovinos fresco e água, na proporção de $50 \% \mathrm{v} / \mathrm{v}$, por um período de 21 dias, em recipiente plásticos e na ausência de ar. No sistema convencional, além de esterco bovino, foi utilizado o fertilizante NPK (10:10:20), após 15, 30 e 45 dias.

\subsection{Coleta das amostras}

Coletaram-se ao acaso nas hortas orgânica e convencional, cinco amostras de alface, rúcula e almeirão, em ponto de maturação comercial, ou seja, aproximadamente 32 dias após o transplante. As hortaliças foram encaminhadas imediatamente para o Centro de Ciência e Tecnologia de Alimentos da UNIDERP para a elaboração dos extratos metanólicos empregados na avaliação do potencial antioxidante e quantificação de fenólicos.

\subsection{Obtenção do extrato metanólico}

As hortaliças in natura (50 g) previamente fracionadas foram maceradas por seis dias utilizando-se metanol 70\% como solvente. Os extratos obtidos foram concentrados em rotavapor sob pressão reduzida e temperatura inferior a $50^{\circ} \mathrm{C}$ e o volume final aferido para $50 \mathrm{~mL}$ com metanol. A partir dos extratos metanólicos foram feitas diluições para obtenção das soluções testes nas concentrações de 0,$01 ; 0,1 ; 1$ e 10 mg.mL $\mathrm{mL}^{-1}$.

\subsection{Determinação da atividade antioxidante}

A atividade antioxidante das hortaliças orgânicas e convencionais analisadas neste estudo foram mensuradas de acordo com o método da redução do radical livre DPPH, descrito por Koleva et al. (2002) com modificações propostas por Choi et al. (2002). Assim, alíquotas de 2,5 mL dos extratos das hortaliças orgânicas e convencionais nas concentrações de 0,01 a $10 \mathrm{mg} \cdot \mathrm{mL}^{-1}$ foram tratadas com $1 \mathrm{~mL}$ de solução metanólica de DPPH 0,3 mM. Após agitação, os tubos foram deixados em repouso ao abrigo da luz por 30 minutos. Decorrido o tempo de reação, a absorbância das amostras foi obtida em $518 \mathrm{~nm}$ contra um branco específico para cada concentração $(2,5 \mathrm{~mL}$ de extrato e 1,0 mL de metanol). A capacidade de sequestrar o radical livre foi expressa de duas formas: através do valor da concentração inibitória $\left(\mathrm{IC}_{50}\right)$, que representa a quantidade de substância antioxidante necessária para reduzir em $50 \%$ a concentração inicial de DPPH e através do percentual de inibição de oxidação do radical calculado segundo a Equação 1:

$\%$ de Redução $($ DPPH consumido $)=\frac{\text { Abs. controle }- \text { Abs. amostra }}{\text { Abs. controle }} \times 100$

\subsection{Determinação de fenólicos totais}

O teor de fenólicos totais dos extratos metanólicos das hortaliças orgânicas e convencionais foi determinado através do método espectrofotométrico, utilizando o reagente de 
Folin-Ciocalteau (SWAUN; HILLIS, 1959; WETTASINGLE; SHAHIDI, 1999) e curva padrão de ácido gálico (EAG). Os resultados foram expressos em $\mathrm{mg}$ de fenólicos totais em equivalente de ácido gálico (EAG) por $100 \mathrm{~g}$ de hortaliça.

\subsection{Análise estatística}

Os dados obtidos foram analisados estatisticamente pelo programa GraphPad Instat, versão 3.06. A significância estatística foi estudada através da análise de variância (ANOVA) e teste de Tukey $(\mathrm{p} \leq 0,05)$.

\section{Resultados e discussão}

O ensaio de redução do radical livre DPPH constitui um método químico frequentemente utilizado para a investigação do potencial antioxidante de extratos de vegetais (GUO et al., 2001; SANDOVAL et al., 2002; LLORACH et al., 2003) e possibilita variação no modo de apresentação dos resultados concernentes à atividade antioxidante investigada. Assim, os resultados deste trabalho foram expressos como a capacidade de sequestrar/reduzir o radical DPPH em porcentagem (Figura 1) e pelo valor de IC50 (Tabela 1), que é um parâmetro indicativo da concentração inibitória necessária para diminuir em 50\% o radical livre DPPH (IC50) (EL; KARAKAYA, 2004; QIAN; NIHORIMBERE, 2004).

Em relação à capacidade de sequestrar o radical livre DPPH, expressa como o percentual de inibição (\% DPPH consumido) dos extratos das hortaliças orgânicas e convencionais analisadas, verificou-se que, quando avaliados individualmente, a ação antioxidante foi significativamente maior nos extratos mais concentrados. No entanto, ao comparar as diferentes concentrações dos extratos das hortaliças orgânicas frente às convencionais, houve variação na intensidade do efeito antioxidante.

Os extratos de alface orgânica e convencional analisados neste estudo reduziram na concentração de $1 \mathrm{mg} \cdot \mathrm{mL}^{-1}$, respectivamente, $72,3 \pm 0,93 \%$ e $62,7 \pm 0,51 \%$ do $\mathrm{DPPH}$, possuindo capacidade antioxidante superior aos extratos analisados por Melo et al. (2006), uma vez que estes autores relataram redução de 36 e 50\% do DPPH nos extratos metanólicos de alface crespa e lisa, respectivamente.

Matsuura et al. (2003) relataram, na concentração de $1 \mathrm{mg} \cdot \mathrm{mL}^{-1}$, redução de apenas 20\% do DPPH nas amostras de rúcula analisadas. Resultados semelhantes foram obtidos por Pieroni et al. (2002), ao analisarem extratos etanólicos de rúcula na concentração de 2,5 mg.mL $\mathrm{mL}^{-1}$. Comparando esses dados com os obtidos neste trabalho, observou-se que, independente do tipo de cultivo, as amostras de rúcula foram mais efetivas, uma vez que reduziram na concentração de $1 \mathrm{mg} \cdot \mathrm{mL}^{-1}$ mais de $70 \%$ o DPPH inicial.

Tabela 1. Atividade antioxidante dos extratos metanólicos das hortaliças provenientes de cultivos orgânico e convencional.

\begin{tabular}{lc}
\hline Hortaliças & $\begin{array}{c}\text { Concentração inibitória }\left(\mathrm{IC}_{50}\right) \\
\mathrm{mg} \cdot \mathrm{mL}^{-1}\end{array}$ \\
\hline Rúcula orgânica & 0,60 \\
Almeirão orgânico & 0,62 \\
Alface orgânico & 0,64 \\
Rúcula convencional & 0,68 \\
Almeirão convencional & 0,71 \\
Alface convencional & 0,77 \\
Vitamina C & 0,03 \\
\hline
\end{tabular}

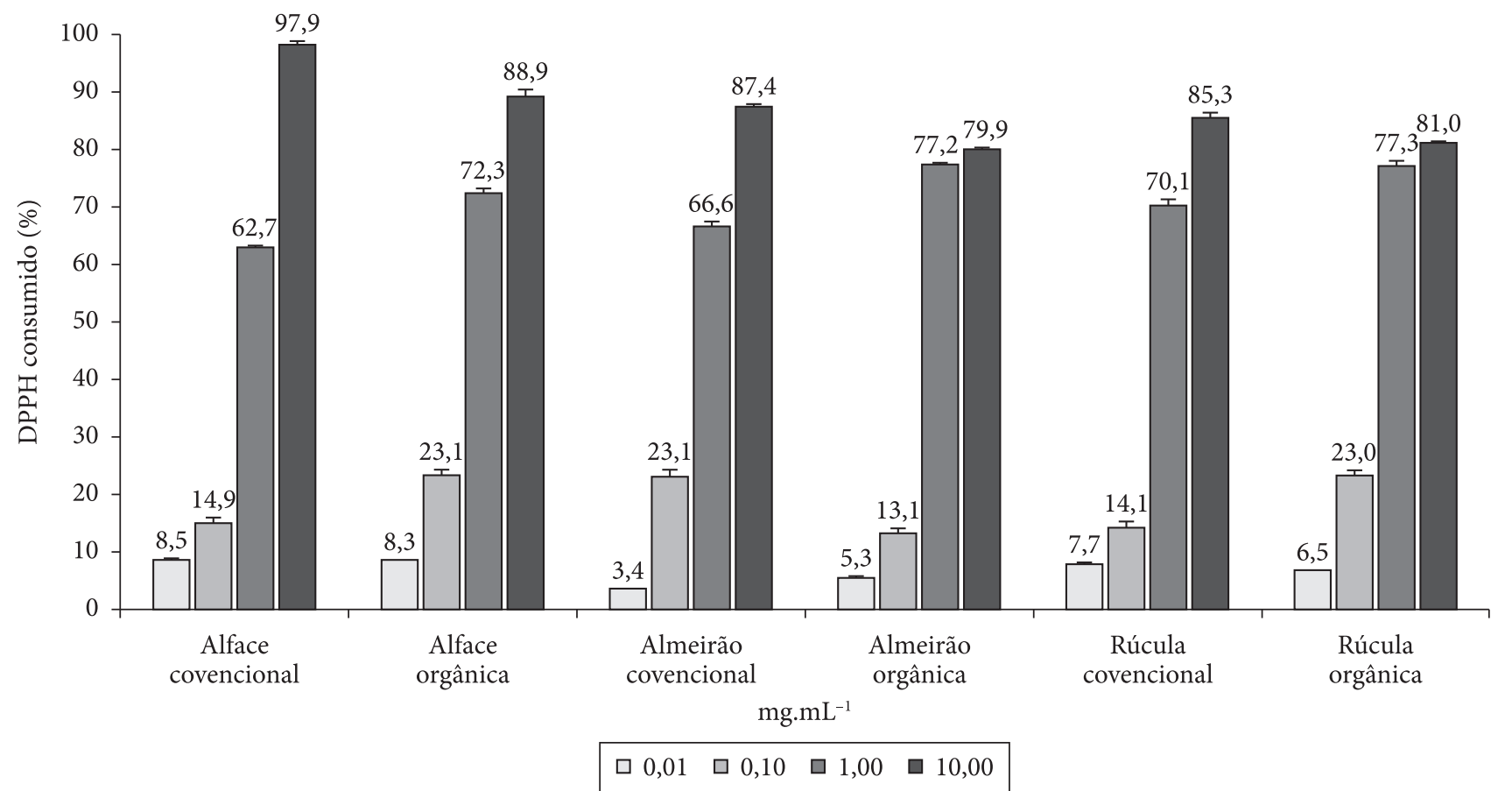

Figura 1. Avaliação da capacidade antioxidante dos extrato das hortaliças pelo método de redução do radical DPPH. 
A atividade antioxidante obtida pelos extratos de almeirão orgânico $(13,1 \pm 0,20 \%)$ e convencional $(23,1 \pm 0,25 \%)$ foi pelo menos duas vezes superior ao efeito obtido pelos extratos desta mesma hortaliça, na concentração de $0,1 \mathrm{mg} \cdot \mathrm{mL}^{-1}$, analisados por El e Karakaya (2004), os quais encontraram redução de apenas 5,95\% do radical livre DPPH. Por outro lado, extratos de almeirão mais concentrados $\left(2,5 \mathrm{mg} \cdot \mathrm{mL}^{-1}\right)$, avaliados por Pieroni et al. (2002), foram capazes de reduzir em cerca de 30\% o radical livre.

De maneira geral, a alface orgânica apresentou ação antioxidante mais efetiva que a convencional nas concentrações de 0,1 e $1 \mathrm{mg} \cdot \mathrm{mL}^{-1}$, reduzindo $23,1 \pm 0,35 \%$ e $72,3 \pm 0,93 \%$ o $\mathrm{DPPH}$. Essas concentrações foram também as mais efetivas nos extratos de rúcula orgânica, quando comparado à convencional, reduzindo $23 \pm 0,85 \%$ o DPPH na concentração $0,1 \mathrm{mg} \cdot \mathrm{mL}^{-1} \mathrm{e}$ $77,3 \pm 0,74 \%$ na concentração $1 \mathrm{mg} \cdot \mathrm{mL}^{-1}$. Em relação aos extratos de almeirão orgânico, a atividade antioxidante foi superior nos extratos menos concentrados, 0,01 e $0,1 \mathrm{mg} \cdot \mathrm{mL}^{-1}$, reduzindo o DPPH em 5,3 $\pm 0,20 \%$ e $13,1 \pm 0,20 \%$, respectivamente.

Foi possível verificar que, na concentração de $1 \mathrm{mg} \cdot \mathrm{mL}^{-1}$, todos os extratos das hortaliças orgânicas foram significativamente mais efetivos $(\mathrm{p} \leq 0,05)$ na redução do $\mathrm{DPPH}$, quando comparados aos respectivos extratos obtidos na cultura convencional, conforme demonstrado na Figura 1, reduzindo em mais de $70 \%$ o DPPH.

Devido à variação no efeito antioxidante dos extratos das hortaliças em função das concentrações empregadas no ensaio, frequentemente os resultados são apresentados através do valor de IC50, possibilitando uma melhor avaliação, uma vez que esse parâmetro indica a concentração da amostra necessária para reduzir em 50\% o DPPH. Quanto menor o valor de IC50, maior a atividade antioxidante da hortaliça.

Os valores de IC50 obtidos pelas hortaliças orgânicas e convencionais estão apresentados na Tabela 1.

Dentre as hortaliças analisadas, a efetividade na capacidade antioxidante foi, em ordem decrescente, obtida pela rúcula orgânica (IC50 de 0,60 mg.mL $\mathrm{mL}^{-1}$ ), almeirão orgânico (IC50 de $0,62 \mathrm{mg} \cdot \mathrm{mL}^{-1}$ ), alface orgânica (IC50 de $0,64 \mathrm{mg} \cdot \mathrm{mL}^{-1}$ ), rúcula convencional (IC50 de 0,68 mg.mL $\mathrm{m}^{-1}$ ), almeirão convencional (IC50 de $0,71 \mathrm{mg} \cdot \mathrm{mL}^{-1}$ ) e alface convencional (IC50 de $\left.0,77 \mathrm{mg} \cdot \mathrm{mL}^{-1}\right)$, hortaliça esta com a menor ação antioxidante.

Ainda que a atividade antioxidante das hortaliças provenientes de cultivo orgânico tenha sido superior às convencionais, uma vez que apresentaram menores valores para o parâmetro de IC50, ainda assim essa ação foi inferior à atividade antioxidante exercida pelo padrão de vitamina $\mathrm{C}$ (IC50 de 0,03 mg. $\mathrm{mL}^{-1}$ ). Isso pode ser justificado em virtude da vitamina $\mathrm{C}$ ser um padrão purificado e que os extratos das hortaliças apresentam inúmeras substâncias que contribuem para o efeito antioxidante, tais como compostos fenólicos, carotenoides e a própria ação da vitamina $\mathrm{C}$.

Considerando-se o conjunto dos resultados, todas as hortaliças estudadas apresentaram ação antioxidante, entretanto a intensidade dessa ação foi diferenciada, tanto entre as espécies de hortaliças como em função do tipo de cultivo.
Os teores de fenólicos da rúcula, alface e almeirão, provenientes das hortas orgânica e convencional, foram quantificados, uma vez que é reconhecido que as propriedades benéficas das hortaliças se devem, preponderantemente, à presença desses compostos (CAO; SOFIC; PRIOR, 1996; GUO et al., 2001; MENSOR et al., 2001; KOLEVA et al., 2002; EL; KARAKAYA, 2004).

O teor de compostos fenólicos dos extratos das hortaliças, em ordem decrescente, foi: rúcula orgânica $\left(126,84 \pm 4,46 \mathrm{mg}\right.$ EAG.100 $\left.\mathrm{g}^{-1}\right)$, alface orgânica $\left(108,72 \pm 2,34 \mathrm{mg}\right.$ EAG.100 $\left.\mathrm{g}^{-1}\right)$, almeirão orgânico $\left(92,15 \pm 1,09 \mathrm{mg}\right.$ EAG.100 $\left.\mathrm{g}^{-1}\right)$, alface convencional $\left(91,22 \pm 0,91 \mathrm{mg}\right.$ EAG.100 $\left.\mathrm{g}^{-1}\right)$, rúcula convencional $\left(90,78 \pm 2,23 \mathrm{mg}\right.$ EAG.100 $\left.\mathrm{g}^{-1}\right)$ e almeirão convencional $\left(81,04 \pm 3,64\right.$ mg EAG. $\left.100 \mathrm{~g}^{-1}\right)$.

Devido ao limitado número de estudos disponíveis, o enfoque principal na quantificação dos compostos fenólicos dos extratos avaliados foi o de verificar a influência do tipo de cultivo no teor dessas substâncias reconhecidas pela sua ação protetora ao organismo humano. De acordo com os resultados obtidos (Tabela 2), pode-se verificar que os extratos das hortaliças provenientes de cultivo orgânico apresentaram teores de compostos fenólicos superiores significativamente $(\mathrm{p} \leq 0,05)$ aos extratos obtidos das hortaliças convencionais.

Marinova, Ribarova e Atanassova (2005) quantificaram em extratos de rúcula $160 \mathrm{mg} .100 \mathrm{~g}^{-1}$ de compostos fenólicos e em alface teores de 116,2 mg.100 g ${ }^{-1}$, valores semelhantes aos determinados neste estudo.

Apesar de os extratos de almeirão orgânico e convencional terem apresentado os menores teores de compostos fenólicos entre as hortaliças avaliadas, esse resultado foi superior ao encontrado por Aqilk, Ahmad e Mehmood (2006).

Melo et al. (2006) analisaram a capacidade antioxidante e o teor de fenólicos de 14 hortaliças adquiridas em São Paulo - Brasil, dentre elas, as amostras de alface crespa e lisa apresentaram teores de compostos fenólicos de 22,6 e 13,85 mg.100 g $\mathrm{g}^{-1}$, respectivamente.

O teor dos compostos fenólicos e demais fitoquímicos presentes nas frutas e hortaliças é amplamente influenciado por fatores genéticos, condições ambientais, tipo de cultivo, além do grau de maturação e variedade da planta, entre outros (KAHKONEN et al., 1999; KAUR; KAPOOR, 2001; KOLEVA et al., 2002; MELO et al., 2006).

Tabela 2. Teor de fenólicos totais, expresso em mg de equivalente de ácido gálico EAG.100 g-1 de hortaliça.

\begin{tabular}{lcc}
\hline Hortaliças & $\begin{array}{c}\text { Extratos } \\
\text { hortaliças orgânicas }\end{array}$ & $\begin{array}{c}\text { Extratos } \\
\text { hortaliças convencionais }\end{array}$ \\
\hline Rúcula & $126,84 \pm 4,46^{\mathrm{a}, \mathrm{A}}$ & $90,78 \pm 2,23^{\mathrm{b}, \mathrm{D}}$ \\
Alface & $108,72 \pm 2,34^{\mathrm{c}, \mathrm{B}}$ & $91,22 \pm 0,91^{\mathrm{d}, \mathrm{D}}$ \\
Almeirão & $92,15 \pm 1,09^{\mathrm{e}, \mathrm{C}}$ & $81,04 \pm 3,64^{\mathrm{f}, \mathrm{E}}$ \\
\hline
\end{tabular}

Os valores representam média \pm desvio padrão obtidos por meio de três determinações de compostos fenólicos totais; valores com letras minúsculas diferentes na mesma linha indicam que houve diferença estatisticamente significativa entre as médias ao nível de $5 \%$; e valores com letras maiúsculas diferentes na mesma coluna indicam que houve diferença estatisticamente significativa entre as médias ao nível de $5 \%$. 
Os extratos metanólicos de hortaliças orgânicas coletadas no ponto de maturidade comercial apresentaram superioridade no teor de compostos fenólicos em relação aos extratos metanólicos obtidos das mesmas hortaliças em cultivo convencional.

\section{Conclusões}

Embora tenha sido demonstrada variação no efeito antioxidante das hortaliças estudadas em função da concentração testada, ficou evidente a superioridade das hortaliças provenientes do cultivo orgânico, quando comparadas às obtidas no sistema convencional.

Verificou-se que o sistema empregado no cultivo de hortaliças contribui, concomitante com o maior teor de compostos fenólicos, para uma mais efetiva atividade antioxidante das hortaliças orgânicas.

\section{Referências bibliográficas}

AQILK, F.; AHMAD, I.; MEHMOOD, Z. Antioxidant and free radical scavenging properties of twelve traditionally used indian medicinal plants. Turkish Journal of Biology, v. 30, n. 3, p. 177-183, 2006.

BOURN, D.; PRESCOTT, J. A comparasion of the nutritional value, sensory qualities, and food safety of organically and conventionally produced food. Critical Reviews in Food Science and Nutrition, v. 42, n. 1, p. 1-34, 2002.

BUAINAIN, A. M.; BATALHA, M. O. (Coord.). Cadeia produtiva de produtos orgânicos. Brasília: MAPA/SPA, 2007. 108 p. (Série Agronegócios, v. 5).

CAI, Y. et al. Antioxidant activity and phenolic compounds of 112 traditional Chinese medicinal plants associated with anticancer. Life Sciences, v. 74, n. 17, p. 2157-2184, 2004.

CAMPOS, M. C. Territorialização da agricultura orgânica no Paraná: preservando o meio ambiente e produzindo alimentos sadios. Disponível em: <http://www.igeo.uerj.br/VICBG-2004/Eixo1/ e1\%20279.htm>. Acesso em: 18 abril 2005.

CAO, G.; SOFIC, E.; PRIOR, R. L. Antioxidant capacity of tea and common vegetable. Journal of Agricultural Food Chemistry, v. 44, n. 11, p. 3426-3431, 1996.

CHOI, C. W. et al. Antioxidant activity and free radical scavenging capacity between korean medicinal plants and flavonoids by assay-guided comparison. Plant Science, v. 163, n. 6, p. 1161-1168, 2002.

DAROLT, M. R. Comparação entre a qualidade do alimento orgânico e a do convencional. In: STRINGHETA, P. C.; MUNIZ, J. N. Alimentos orgânicos: produção, tecnologia e certificação. Viçosa: Editora UFV, p. 289-312, 2003a.

DAROLT, M. R. A qualidade dos alimentos orgânicos. 2003b. Disponível em: <http://www.planetaorgânico.com.br/daroltqualid. htm>. Acesso em: 19 setembro 2003.

EL, S. N.; KARAKAYA, S. Radical scavenging and iron-chelating activities of some greens used as traditional dishes in Mediterranean diet. International Journal of Food Science Nutrition, v. 55, n. 1, p. 67-74, 2004.

GUO, J. et al. Antioxidant properties of the extracts from different parts of broccoli in Taiwan. Journal of Food Drug Analysis, v. 9, n. 2, p. 96-101, 2001.

HSU, C. Y.; CHAN, Y. P.; CHANG, J. Antioxidant activity of extract from Polygonum cuspidatum. Biological Research, v. 40, p.13-21, 2007.
KAHKONEN, M. P. et al. Antioxidant activity of plant extracts containing phenolic compounds. Journal Agricultural Food Chemistrry, v. 47, n. 10, p. 3954-3962, 1999.

KAUR, C.; KAPOOR, H. C. Antioxidants in fruits and vegetables the millennium's health. International Journal Food Science Technology, v. 36, n. 7, p. 703-725, 2001.

KOLEVA, L. I. et al. Screening of plant extracts for antioxidant activity: a comparative study on three testing methods. Phytochemical Analysis, v. 13, n. 1, p. 8-17, 2002.

LLORACH, R. et al. Valorization of cauliflower (Brassica oleracea L. var. botrytis) by-products as a source of antioxidant phenolics. Journal Agricultural Food Chemistry, v. 51, n. 8, p. 2181-2187, 2003.

MACHADO, F.; CORAZZA, R. Desafios tecnológicos, organizacionais e financeiros da agricultura orgânica no Brasil. Revista de la Facultad de Economia, v. 26, n. 4, p. 21-40, 2004.

MAGKOS, F.; ARVANITI, F.; ZAMPELAS, A. Organic food: nutritious food or food for thought? A review of the evidence. International Journal Food Science Nutrition, v. 54, n. 5, p. 357-71, 2006.

MARINOVA, D.; RIBAROVA, F.; ATANASSOVA, M. Total phenolics and total flavonoids in bulgarian fruits and vegetables. Journal of the University of Chemical Technology and Metallurgy, v. 40, n. 3, p. 255-260, 2005.

MATSUURA, $\mathrm{H}$. et al. DPPH radical scavengers from dried leaves of oregano (Origanum vulgare). Bioscience Biotechnology Biochemistry, v. 67, n. 11, p. 2311-2316, 2003.

MELO, E. A. et al. Capacidade antioxidante de hortaliças usualmente consumidas. Ciência Tecnologia de Alimentos, v. 26, n. 3, p. 639-644, 2006.

MENSOR, L. L. et al. Screening of brasilian plant extracts for antioxidant activity by the use of DPPH free radical method. Phytotherapy Research, v. 15, n. 2, p. 127-130, 2001.

MIGUEL, G. et al. Composition and antioxidant activities of the essential oils of Thymus caespititius, Thymus camphoratus and Thymus mastichina Food Chemistry, v. 86, n. 2, p. 183-188, 2004.

ORMOND, J. G. P. et al. Agricultura orgânica: quando o passado é futuro. Rio de Janeiro: BNDES, 2002. 34 p.

PENTEADO, S. R. Introdução à agricultura orgânica. Campinas: Grafimagem, 2000.

PIERONI, A. et al. In vitro antioxidant activity of non-cultivated vegetables of ethnic albanians in southern Italy. Phytotherapy Research, v. 16, n. 5, p. 467-473, 2002.

PRIMAVESI, A. Manejo ecológico de pragas e doenças. São Paulo: Nobel, 1988. $137 \mathrm{p}$

QIAN, H.; NIHORIMBERE, V. Antioxidant power of phytochemicals from Psidium guajava leaf. Journal of Zhejiang University Science, v. 5, n. 6, p. 676-683, 2004.

RAMOS, A. et al. Screening of antimutagenicity via antioxidant activity in Cuban medicinal plants. Journal Ethnopharmacol, v. 87, n. 2-3, p. 241-246, 2003.

RICE-EVANS, C. A.; MILLER, N. J.; PAGANGA, G. Structureantioxidant activity relationships of flavonoids and phenolic acids. Free Radical Biology Medicine, v. 20, n. 7, p. 933-956, 1996.

SANDOVAL, M. et al. Antioxidant activity of the cruciferous vegetable Maca (Lipidium meyenii). Food Chemistry, v. 79, n. 2, p. 207-213, 2002

SCHAFFER, S.; SCHMITT-SCHILLIG, S.; ECKERT, G. P. Antioxidant properties of mediterranean food plant extracts: geographical differences. Journal of Physiology and Pharmacology, v. 56, n. 1, p. 115-124, 2005. 
STAUB, G. A. O financiamento do banco do Brasil à agricultura orgânica e preservação ambiental do estado do Paraná. 2003. 139 f. Dissertação - (Mestrado) - Universidade Federal de Santa Catarina, Florianópolis, 2003.

STERTZ, S. C.; ROSA, M. I. S.; FREITAS, R. J. S. Qualidade nutricional e contaminantes da batata (Solanum tuberosum 1., Solanaceae) convencional e orgânica na região metropolitana de Curitiba Paraná. Boletim do CEPPA, v. 23, n. 2, p. 383-396, 2005.

SWAUN, T.; HILLIS, W. E. The phenolic constituents of Prunus domestica. The quantitative analysis of phenolic constituents. Journal Science Food Agriciltural, v. 10, p. 63-68, 1959.

VELIOGLU, Y. S. et al. Antioxidant activity and total phenolics in selected fruits, vegetables and grain products. Journal Agricultural Food Chemistry, v. 46, n. 10, p. 4113-4117, 1998.

VISON, J. A. et al. Phenol antioxidant quantity and quality in foods: vegetables. Journal Agricultural Food Chemistry, v. 46, n. 9, p. 3630-3634, 1998.
WANG, H.; CAO, G.; PRIOR, R. L. Total antioxidant capacity of fruit. Journal Agricultural Food Chemistry, v. 44, p. 701-705, 1996.

WETTASINGHE, M.; SHAHIDI, F. Evening primrose meal: a source of natural antioxidants and scavenger of hydrogen peroxide and oxygen-derived free radicals. Journal Agricultural Food Chemistry, v. 47, n. 5, p. $1801-1812,1999$.

WILLIAMS, C. M. Nutritional quality of organic food: shades of grey or shades of green? Proceedings of the Nutrition Society, v. 61, n. 1, p. 19-24, 2002.

WORTHINGTON, V. Nutritional quality of organic versus conventional fruits, vegetable and grains. The Journal of Alternative Complementary Medicine, v. 7, n. 2, p. 161-173, 2001.

YU, F. et al. Antioxidant activities of crude tea polyphenols, polysaccharides and proteins of selenium-enriched tea and regular green tea. European Food Research and Technology, v. 225, n. 5-6, p. 843-848, 2007. 